\title{
System Independent Fault Diagnosis for Synchronous Generator
}

\author{
Jeet Gandhi ${ }^{1}$, R. Gopinath ${ }^{2}$, C. Santhosh Kumar ${ }^{3}$ \\ 1,2,3 Machine Intelligence Research Laboratory, \\ Department of Electronics and Communication Engineering, \\ Amrita School of Engineering, Coimbatore, \\ Amrita Vishwa Vidyapeetham, Amrita University, India-641112 \\ jeetgandhi12@gmail.com \\ rgopinath.gct@gmail.com \\ cs_kumar@cb.amrita.edu
}

\begin{abstract}
Creating a unified fault diagnosis model that can detect faults across systems with different ratings (system independent fault diagnosis) would be of great interest in making condition-based maintenance (CBM) more popular. In this work, three phase synchronous generators with 3 and $5 \mathrm{kVA}$ ratings are used for detecting stator inter-turn short circuit faults.

Our baseline is a $3 \mathrm{kVA}$ generator working at $1 \mathrm{~A}$ load during training and testing, to emulate the system/load dependent fault diagnosis. We obtained a classification accuracy of $99.75 \%, 100 \%$ and $100 \%$ for R phase, $\mathrm{Y}$ phase and B phase faults respectively. Subsequently, we evaluated the system for its load independent performance. Performance accuracy deteriorated due to the load specific variations (LSV) in the input feature vector (IFV). LSV is undesired, and we used nuisance attribute projection (NAP) to remove them. Using NAP, we obtained a performance improvement of $23.13 \%, 17.75 \%$ and $20.72 \%$ for three fault models on the $3 \mathrm{kVA}$ generator and similar performance improvement was obtained for $5 \mathrm{kVA}$ generator also.
\end{abstract}

Further, we experimented for load and system independent fault diagnosis. In this case, we consider LSV and system specific variations (SSV) on IFV as undesired. We experimented with two types of NAP, (1) single step NAP, (2) stacked NAP. Experimental results show that the two staged stacked NAP outperforms. We obtained an improvement of

Jeet Gandhi et al. This is an open-access article distributed under the terms of the Creative Commons Attribution 3.0 United States License, which permits unrestricted use, distribution, and reproduction in any medium, provided the original author and source are credited.
$23.99 \%, 16.06 \%$ and $28.39 \%$, in classification accuracy for three fault models, resulting in overall classification accuracy of $89.22 \%, 94.67 \%$ and $94.59 \%$ for $\mathrm{R}$ phase, $\mathrm{Y}$ phase and $\mathrm{B}$ phase fault models respectively.

\section{INTRODUCTION}

Condition-based maintenance (CBM) is becoming increasingly important due to its ability to reduce maintenance cost and improve productivity (Jardine, Lin, \& Banjevic, 2006). It involves continuously monitoring of system condition by observing different parameters, and maintenance decision is taken if machine starts behaving abnormally. Fault diagnosis is an integral part of CBM, which involves fault detection, fault isolation and fault identification.

CBM can be performed using three approaches: model based, data driven and hybrid approaches. Model based approach involves mathematical modeling of a physical system, hence it requires complete knowledge of the system design and its specifications. For complex systems, it is very difficult to implement mathematical model incorporating all design specifications, hence model based approach is not very popular for such systems. Data driven approach does not involve modeling of the system, hence complete knowledge of system design and its specifications is not required (Yin, Ding, Xie, \& Luo, 2014). It involves recording of system parameters during good and faulty conditions, and analysis of recorded signals to determine fault signatures.

Fault diagnosis of synchronous generator has been explored by many researchers to date. Nanidi et al. (Nandi, Toliyat, $\& \mathrm{Li}, 2005)$ described various types of faults generally occurring in electrical machines, out of which almost $30 \%$ to 
$40 \%$ faults are stator winding related faults. Stator winding short circuit faults develop from inter-turn faults, which occurs mainly because of insulation failure. If not addressed, inter-turn faults increase winding temperature and lead to insulation failure, which may result in coil to coil shorting, and even entire machine could breakdown. Hence prior diagnosis of inter-turn faults is very important for condition based monitoring of synchronous generators (Siddique, Yadava, \& Singh, 2005). Siddique et al. (Siddique et al., 2005) illustrated various monitoring techniques for stator winding fault diagnosis of induction machines. Among all monitoring techniques, motor current is most widely used, because current sensors are already available in most machines and measurement is non-invasive. Gandhi et al. (A. Gandhi, Corrigan, $\&$ Parsa, 2011) reviewed recent trends in diagnosis of stator winding inter-turn faults.

Various signal processing techniques have been experimented to analyze recorded signals and to identify distinguishable fault signatures. Neti et al. (Neti \& Nandi, 2009) analyzed different harmonics in stator current and investigated their applicability for fault detection. Since stator current is nonstationary, time resolution is lost while performing Fourier transform. Hence analyzing signal in frequency domain is not sufficient for the fault diagnosis. For such non-stationary signals, Hilbert-Huang Transformation (HHT) or wavelet transform (WT) can be used for better time-frequency representation. Wang et al. (Wang, Liu, \& Chen, 2014) experimented Hilbert-Huang Transformation (HHT) to diagnose incipient stator insulation fault in permanent magnet synchronous generator. Dash et al. (Dash, Subudhi, \& Das, 2010) used discrete wavelet transform (DWT) for analyzing behavior of interturn fault in induction motor.

Artificial intelligence (AI) based techniques have also been experimented to discover knowledge about health of the system, so that diagnosis can be performed with less human intervention (Awadallah \& Morcos, 2003). Bessam et al. (Bessam, Menacer, Boumehraz, \& Cherif, 2017) used stator current signals with wavelet transform and neural network classifier to diagnose and locate inter-turn faults in induction motor. Rajeswari et al. (Rajeswari \& Kamaraj, 2007) used wavelet based adaptive neuro-fuzzy inference system (ANFIS) to detect inter-turn faults in synchronous generator. Gopinath et al. (Krishna et al., 2016; Gopinath, Kumar, Vishnuprasad, \& Ramachandran, 2015) used feature mapping technique with support vector machine (SVM) classifier for inter-turn fault detection in synchronous generators.

From literature survey, it can be found that existing CBM realizations use synchronous generator of specific rating for data collection, and developed diagnosis model is tested on machine with same ratings only. However in factory environment, many machines with different power ratings are generally used, and these approaches require separate diagno- sis model for each machine with different ratings. To overcome this problem, machine independent fault diagnosis system can be used, where single diagnosis model can be used to detect faults in synchronous generators of same characteristics but having different power ratings.

Because of different power ratings and different loading conditions, fault signatures captured by diagnosis model will have system specific variations (SSV) and load specific variations (LSV) (Gopinath, Kumar, \& Ramachandran, 2016). Because of these variations, performance of the fault diagnosis model deteriorates. These variations can be considered as a nuisance for classification (J. Gandhi et al., 2017; Gopinath, Kumar, Ramachandran, Upendranath, \& Kiran, 2016), and their effect on fault signatures should be reduced to have a robust fault diagnosis model. Such nuisance attributes can be projected out from the input feature vector (IFV) using nuisance attribute projection (NAP). NAP was proposed by Solomonoff et al. (Solomonoff, Campbell, \& Quillen, 2007; Solomonoff, Campbell, \& Boardman, 2005) for automatic speaker recognition (ASR) system, where biggest challenges for performance improvement were channel and handset variations. Because of different microphones used and environment noise, there were variations in utterances recorded from same speaker and classification performance was degraded because of these variations. NAP was used to project out such nuisance attributes from original feature space. NAP was also experimented in face recognition system to diminish effect of environmental factors such as illumination (Štruc et al., 2010; Tome et al., 2012; Yifrach et al., 2016).

In this work, we experimented two NAP approaches to reduce multiple nuisance variations from IFV, stacked NAP and single stage NAP (Solomonoff et al., 2007). In stacked NAP approach, SSV can be removed in first layer and LSV can be removed in second layer, or LSV can be removed in first layer and SSV can be removed in second layer. In single stage NAP approach, both LSV and SSV can be removed simultaneously using composite weight matrix. From experiments, we note that by removing SSV in first layer and LSV in second layer in stacked NAP, optimum classification performance can be obtained.

This paper is organized as follows. Section 2 explains experimental setup and design of stator winding for fault injection. Data collection is explained in section 3. Discrete wavelet transform (DWT) and nuisance attribute projection (NAP) are discussed briefly in section 4 . Experiments and results are described in section 5 and section 6 concludes the paper.

\section{EXPERIMENTAL SETUP}

Normally in synchronous generators, taps are provided at $0 \%$ and $100 \%$ of winding coils in stator and field windings. However more terminals are required to introduce faults for condition based maintenance. In this work, two custom-made syn- 


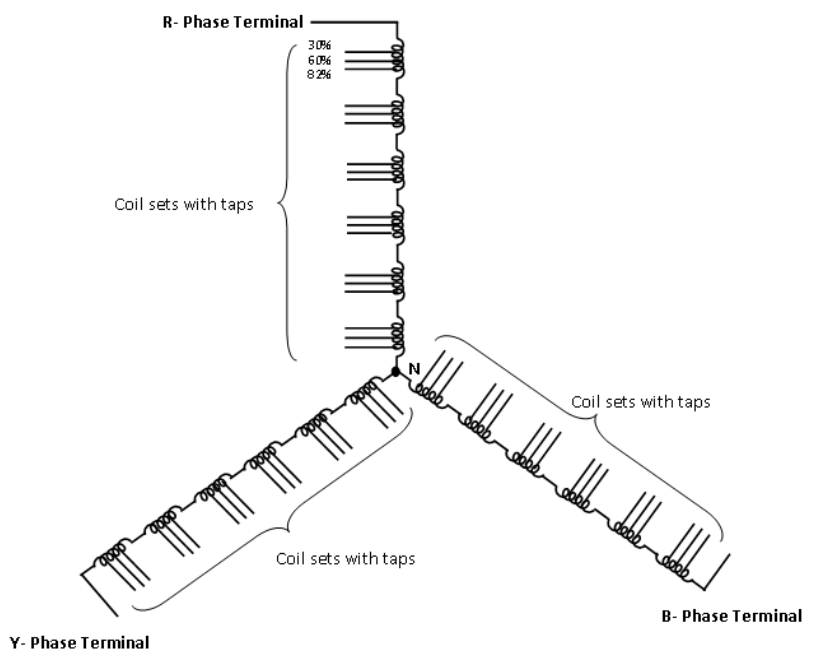

Figure 1. Design of stator winding for fault injection

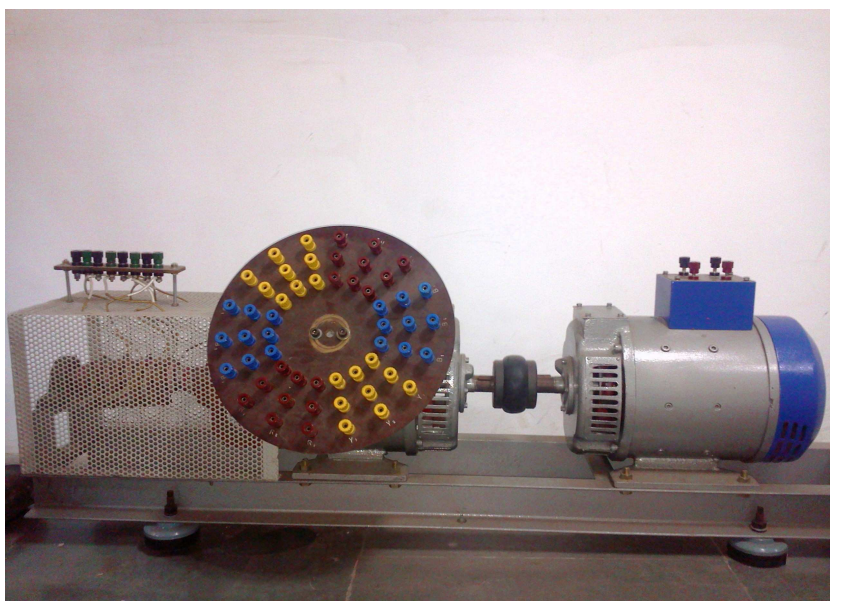

Figure 2. Synchronous generator test setup

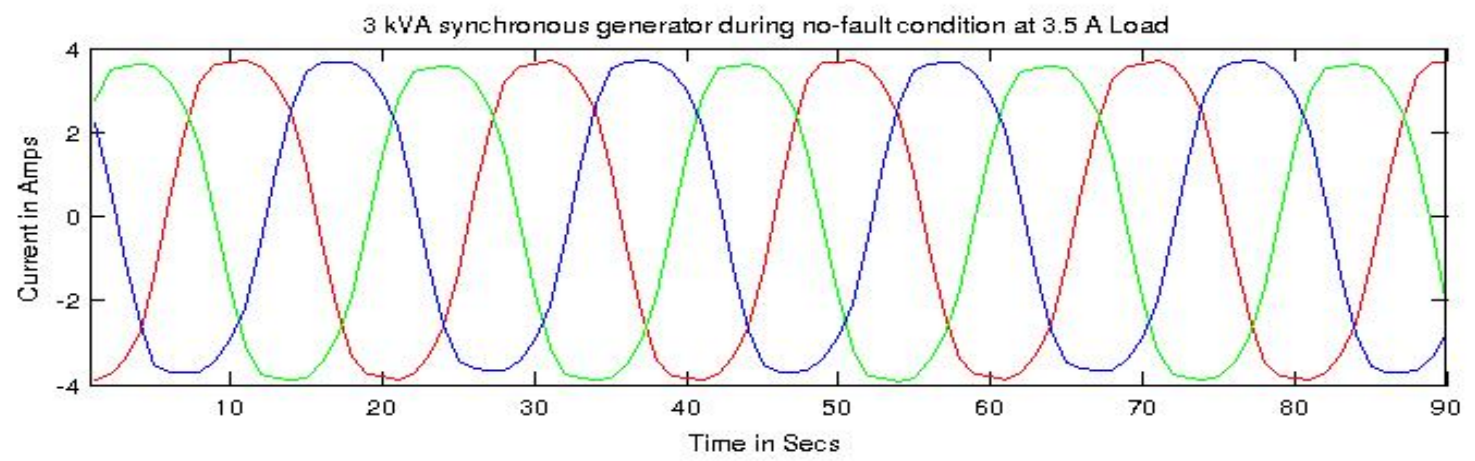

3 kVA synchronous generator during inter-turn fault condition (14 turns shorted) in the R phase of stator winding at 3.5 A Load

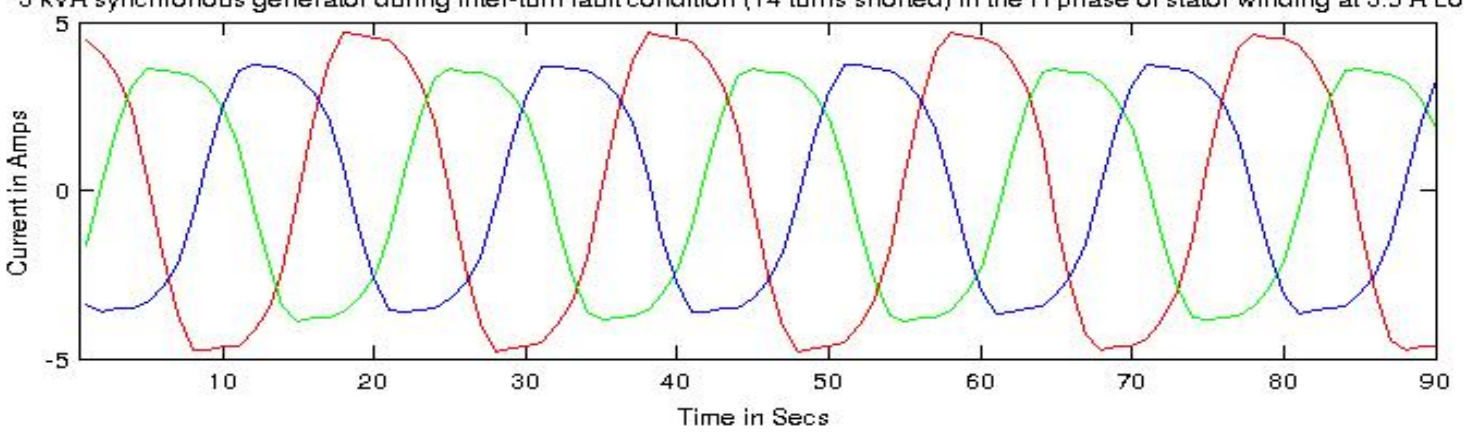

Figure 3. Current signals from the synchronous generator during healthy and fault condition 
chronous generators with power ratings of $3 \mathrm{kVA}$ and $5 \mathrm{kVA}$ are used. Three taps are provided at $30 \%, 60 \%$ and $82 \%$ in each coil and six coils are present in each phase, i.e. R phase, $\mathrm{Y}$ phase and B phase. Hence 18 taps are there for each phase and 54 total taps in stator winding. These taps are accessible from terminal board situated on the machine and can be used to inject inter-turn faults. Design of stator winding is shown in Figure 1 and synchronous generator test setup is shown in Figure 2. Detailed description of test setup is elaborated in (Gopinath et al., 2013).

\section{DATA COLlection}

CBM requires recording of signals during healthy and faulty conditions to understand fault behavior of the system. Interturn faults of different severity are injected through taps provided in each coil of stator winding using terminal board. Since load may change during operation in industrial environment, three phase resistive load bank is used to emulate these variations and experiments are carried out at different loads i.e. 0.5 A, 1 A, 1.5 A, 2 A, 2.5 A, 3 A, 3.5 A. Hall effect current sensors are used with National Instruments (NI) PXI 6221 data acquisition system and LabVIEW SignalExpress software to record stator current signals. In each experiment, machine is operated for 10 seconds and sampling frequency of $1 \mathrm{kHz}$ is used, hence 10000 samples are recorded for each case. We have partitioned recorded samples into different frames of length 512 with overlap of 256 samples. This procedure is carried out for both systems with all working conditions. The acquired current signal from the synchronous generator during healthy and fault condition is shown in the Figure 3.

\section{Methodologies USED}

\subsection{Feature extraction using discrete wavelet transform (DWT)}

For non-stationary signals like stator current, time resolution is lost while performing Fourier transform. For better time and frequency localization, wavelet transform is the best option for time-frequency representation. It is multi-resolution analysis technique, where better frequency localization can be achieved at lower frequencies and better time localization can be achieved at higher frequencies (Yan, Gao, \& Chen, 2014).

Continuous wavelet transform (CWT) involves scaling and shifting of mother wavelet function, in which scale and translation parameters are varied continuously. This results in redundant information and it requires very high computation time. To avoid this, DWT can be used for faster computation in which scale and translation parameters are discretized on dyadic scale. DWT can be performed using filter banks containing high pass and low pass filters. Input signal is passed through filter bank and then down-sampled by 2. One level

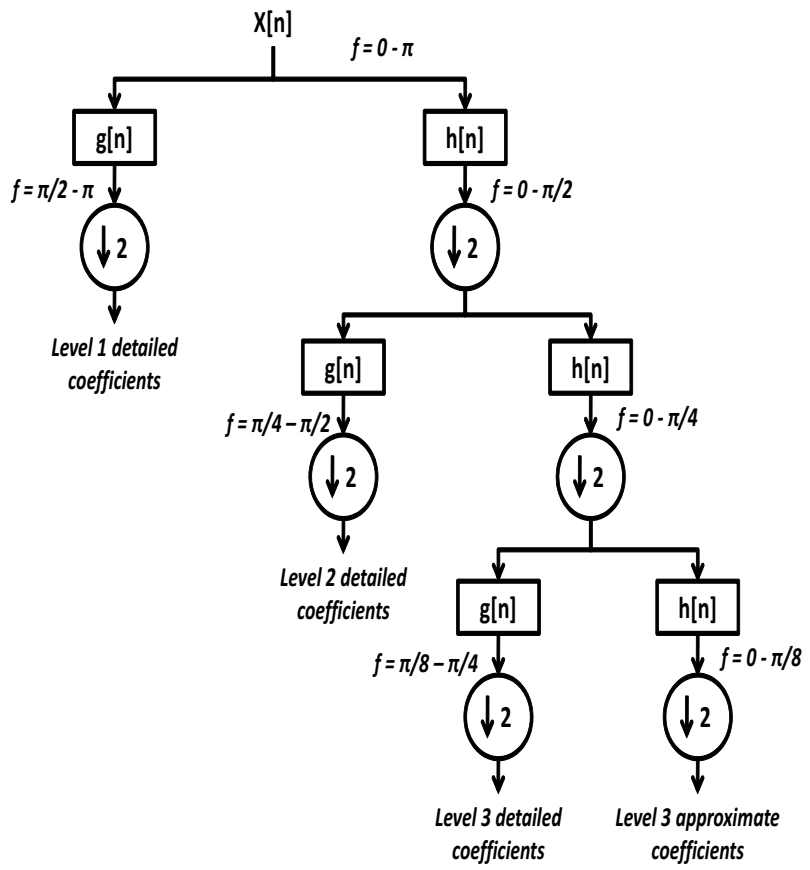

Figure 4. Discrete wavelet transform decomposition

of decomposition is carried out by following this procedure, which can be formulated as

$$
\begin{gathered}
y_{\text {high }}[k]=\sum_{n} x[n] \cdot g[2 k-n] \\
y_{\text {low }}[k]=\sum_{n} x[n] \cdot h[2 k-n]
\end{gathered}
$$

where $y_{\text {high }}$ and $y_{\text {low }}$ represents detailed and approximate coefficients respectively, and $g[n]$ and $h[n]$ represents impulse response of high pass filter and low pass filter respectively. DWT decomposition procedure is illustrated in Figure 4.

Selection of level of decomposition and wavelet function is very crucial for particular application. Minimum level of decomposition depends upon signal frequency $f$ and sampling frequency $f_{s}$ (Dash et al., 2010), which can be formulated as

$$
N_{f}=\text { integer }\left[\frac{\log \left(f_{s} / f\right)}{\log (2)}\right]
$$

For our problem, with signal frequency of $50 \mathrm{~Hz}$ and sampling frequency of $1 \mathrm{kHz}$, calculated minimum level of decomposition is 4 . In this work, we have experimented 4, 5 and 6 levels of decomposition. For machine fault diagnosis, most popular wavelets used are Haar, Daubechies, Symlets, Coiflet and Morlet. Because of its orthonormal bases and smoother function (Daubechies, 1988), daubechies wavelets have been widely experimented in machine fault diagnosis (Bendjama, Bouhouche, \& Boucherit, 2012; Wu \& Liu, 2008). In this work, daubechies family wavelets $\mathrm{db} 2, \mathrm{db} 4, \mathrm{db} 6, \mathrm{db} 8$ and 
db10 were experimented.

To avoid redundancy in coefficients and large computational time, features need to be extracted from wavelet coefficients. Statistical features can be calculated for each level detailed coefficients and for final level approximate coefficients. In this work, following statistical features were calculated (Erişti, Uçar, \& Demir, 2010):

1. Mean $f_{1}=\frac{\sum_{i=1}^{N} c_{i}}{N}$

2. Standard deviation $f_{2}=\sqrt{\frac{\sum_{i=1}^{N}\left(c_{i}-f_{1}\right)^{2}}{(N-1)}}$

3. Skewness $f_{3}=\frac{N}{(N-1)} \sum_{i=1}^{N}\left(\frac{\left(c_{i}-f_{1}\right)^{3}}{f_{2}^{3}}\right)$

4. Kurtosis $f_{4}=\left\{\frac{N(N+1)}{(N-1)(N-2)(N-3)} \sum_{i=1}^{N}\left[\frac{c_{i}-f_{1}}{f_{2}}\right]^{4}\right\}-$ $\frac{3(N-1)^{2}}{(N-2)(N-3)}$

5. $\quad$ RMS $f_{5}=\sqrt{\frac{1}{N} \sum_{i=1}^{N} c_{i}^{2}}$

6. Form Factor $f_{6}=\frac{f_{1}}{f_{5}}$

7. Crest Factor $f_{7}=\frac{p e a k}{f_{5}}$

8. $\quad$ Energy $f_{8}=\sum_{i=1}^{N}\left|c_{i}^{2}\right|$

9. Shannon entropy $f_{9}=-\sum_{i=1}^{N} c_{i}^{2} \log \left(c_{i}^{2}\right)$

10. Log energy entropy $f_{10}=\sum_{i=1}^{N} \log \left(c_{i}^{2}\right)$

11. Interquartile range $f_{11}=c_{75}-c_{25}$ where $c_{i}$ is wavelet coefficient, $c_{25}$ and $c_{75}$ are 25 th and 75 th quantile respectively and $N$ is the length of coefficients.

\subsection{Nuisance attribute projection (NAP)}

Nuisance attribute projection has been extensively used in pattern recognition to reduce influence of nuisance attributes (Solomonoff et al., 2007, 2005; Štruc et al., 2010; Tome et al., 2012; Yifrach et al., 2016). In system independent fault diagnosis, system capacity variations and load variations lead to deviation in fault signatures, which degrades the performance of diagnosis model. These variations can be considered as nuisance attributes, and effect of such nuisance attributes should be reduced for robust fault diagnosis.

One of the simplest way to diminish effect of nuisance attributes is using projection. NAP identifies subspace carrying nuisance attributes and remove such subspace from original feature space. This can be represented pictorially as shown in Figure 5, where $m^{(s, c)}$ indicates feature carrying

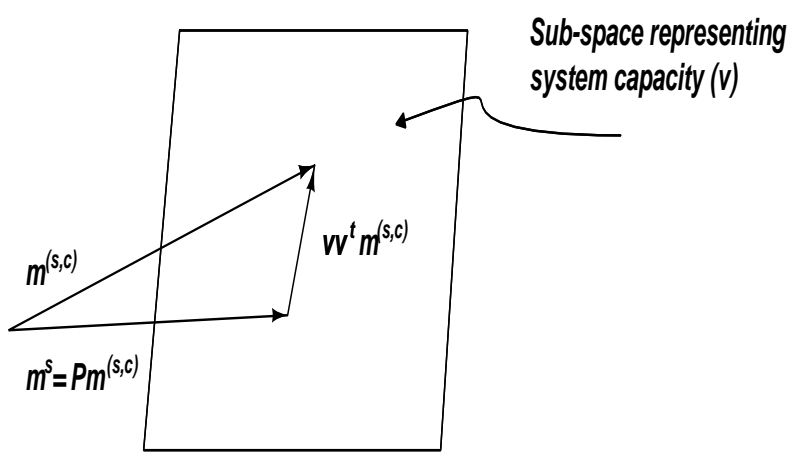

Figure 5. Nuisance attribute projection (NAP)

nuisance variations and $m^{(s)}$ indicates transformed feature without nuisance variations. This projection can be represented mathematically as

$$
P=I-V V^{T}
$$

where $\mathrm{V}$ is the matrix whose columns represent nuisance attributes, and $\mathrm{P}$ is projection matrix to be used for removing nuisance attributes.

In NAP, each training feature vector is labeled with desired attribute and/or target attribute. Weight matrix $\mathrm{W}$ is formulated from these labels, whose entries depict relationship between pairs of training feature vectors. Entry of $\mathrm{W}$ matrix, represented by $W_{i j}$, should be positive if both feature vectors need to be moved together, should be negative if both feature vectors need to be moved apart, and should be zero if there is no relationship between pair of feature vector.

NAP involves minimization of following figure of merit with projection matrix $\mathrm{P}$.

$$
\delta=\sum_{i j} W_{i j}\left\|P\left(x_{i}-x_{j}\right)\right\|^{2}
$$

which can be solved using following eigenvalue problem

$$
A Z A^{T} V=V \Lambda
$$

where $\mathrm{V}$ is a matrix, in which $\mathrm{k}$ most principal eigenvectors are arranged column wise, matrix $Z=\operatorname{diag}(W 1)-W$, and A is training feature matrix. Eq. (6) is called "NAP equation". Projection matrix $\mathrm{P}$ can be found using $\mathrm{V}$ matrix as specified in Eq. (4).

Steps to perform nuisance attribute projection (NAP) are mentioned below:

1. Label training samples with desired attribute and/or target attribute.

2. Create square symmetric matrix $W_{i j}$ using these labels. Different definitions of weight matrix are mentioned in section 5 . 


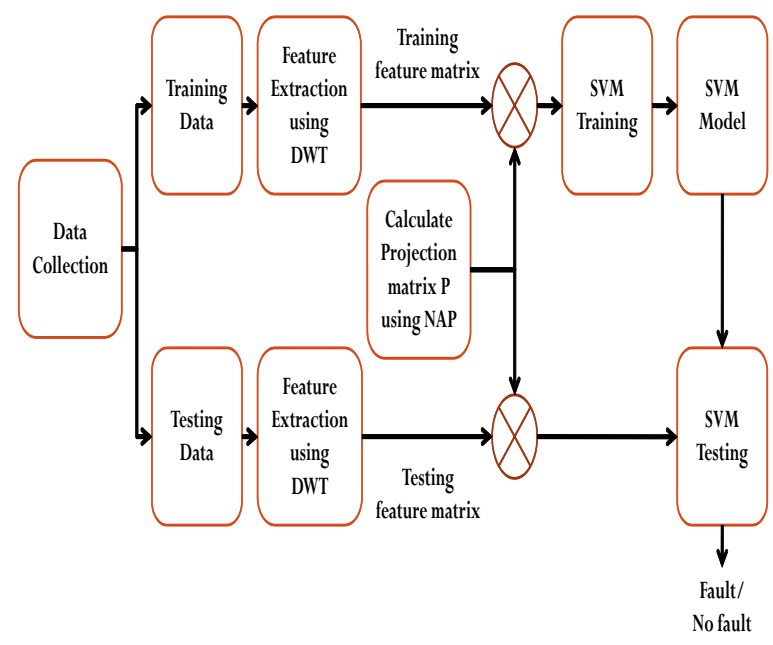

Figure 6. Overall block diagram of proposed approach

3. Calculate matrix $M=A Z A^{T}$, whose eigenvectors represents basis vectors of subspace containing nuisance attributes. Here $A$ is training feature matrix and $Z=$ $\operatorname{diag}(W * 1)-W$.

4. Find eigenvalues and eigenvectors of matrix $M$ and sort them in descending order to find basis vectors for subspace having nuisance attributes.

5. Form matrix $V$ whose columns are $k$ most principal eigenvectors.

6. Calculate projection matrix $P$ using (4), that will project out nuisance attributes.

7. Multiply train feature matrix and test feature matrix with projection matrix to get transformed features having least nuisance variations.

Overall process work flow is explained in Figure 6.

\section{EXPERIMENTAL RESULTS}

We have considered two synchronous generators with power rating of $3 \mathrm{kVA}$ and $5 \mathrm{kVA}$ as two different systems. Three phase stator current signals were recorded in healthy and faulty conditions at different load conditions. Recorded samples of each phase current were partitioned into different frames of length 512. Then we applied DWT on these partitioned frames using db8 mother wavelet and 5 levels of decomposition. After performing DWT, we obtained six coefficient frames which include detailed coefficients of 5 levels and approximate coefficients of the final level. As mentioned in Section 4.1, 11 statistical feature were calculated on each coefficient frames. Hence we obtained 66 statistical features for each phase current. Then we combined these features calculated for all three phase current samples and we obtained 198 statistical features as an output. These 198 features were used as an input to SVM classifier. In all the experiments, we used binary SVM classifier for each phase fault models, i.e. R phase fault vs no fault, Y phase fault vs no fault and B phase fault vs no fault. We used $70 \%$ of recorded samples for training and $30 \%$ of recorded samples for testing. Number of samples used for training and testing for all experiments are mentioned in Table 1.

Table 1. Dataset used for training and testing for all experiments (no-fault data: $55 \%$, fault data: $45 \%$ )

\begin{tabular}{llll}
\hline & Fault & No fault & Total \\
\hline Training data & 13300 & 10640 & 23940 \\
Test data & 5852 & 4683 & 10535 \\
\hline Total & 19152 & 15323 & 34475 \\
\hline
\end{tabular}

The following experiments were performed:

1. Load dependent fault diagnosis

2. Load independent and system dependent fault diagnosis

3. Baseline system for system independent fault diagnosis

4. Removing system specific variations using NAP

5. Removing load specific and system specific variations using stacked NAP

6. Removing load specific and system specific variations using single stage NAP

\subsection{Load dependent fault diagnosis}

In this experiment, current signals were collected at different load conditions for $3 \mathrm{kVA}$ and $5 \mathrm{kVA}$ system. Signals collected for each load and for specific machine were used separately to train and test individual fault diagnosis models. Wavelet domain statistical features were extracted and used with linear support vector machine (SVM) back-end classifier. Classification accuracy of load dependent fault diagnosis models is listed in Table 2. Because of same load condition and system ratings for training and testing, we were able to obtain very good classification performance.

\subsection{Load independent and system dependent fault diag- nosis}

Since load conditions may change during operation in industrial environment, fault diagnosis model should be independent of load changes. In this experiment, we aggregated current signals acquired at different load conditions for each machine separately to emulate load independent behavior. Wavelet domain statistical features and linear support vector machine (SVM) back-end classifier were used to develop baseline models. Classification accuracy of system dependent fault diagnosis baseline models are listed in Table 3.

Extracted features contain LSV, which degrades classifier performance. To remove LSV from IFV, nuisance attribute projection (NAP) was experimented. Definition of weight matrix is mentioned below. 
Table 2. Classification performance of load dependent fault diagnosis models (in \% accuracy)

\begin{tabular}{lllll}
\hline \multirow{2}{*}{ Rating } & \multirow{2}{*}{ Load } & \multicolumn{3}{c}{ Fault } \\
\cline { 3 - 5 } & & $\mathrm{R}$ & $\mathrm{Y}$ & $\mathrm{B}$ \\
\hline & 0.5 & 99.37 & 99.56 & 100 \\
& 1 & 99.75 & 100 & 100 \\
& 1.5 & 100 & 99.94 & 100 \\
$3 \mathrm{kVA}$ & 2 & 99.75 & 100 & 100 \\
& 2.5 & 99.25 & 99.31 & 99.00 \\
& 3 & 100 & 99.94 & 99.94 \\
& 3.5 & 95.99 & 99.50 & 100 \\
\hline & 0.5 & 100 & 99.94 & 99.88 \\
& 1 & 100 & 100 & 100 \\
$5 \mathrm{kVA}$ & 1.5 & 100 & 97.56 & 99.94 \\
& 2 & 99.88 & 99.94 & 98.88 \\
& 2.5 & 98.18 & 99.44 & 98.00 \\
& 3 & 92.04 & 100 & 97.56 \\
& 3.5 & 93.11 & 98.37 & 99.06 \\
\hline
\end{tabular}

$$
W_{i j}= \begin{cases}1, & \text { if class }\left(x_{i}\right)=\operatorname{class}\left(x_{j}\right) \text { and } \\ & \operatorname{load}\left(x_{i}\right) \neq \operatorname{load}\left(x_{j}\right) \\ 0, & \text { otherwise }\end{cases}
$$

Using this weight matrix definition, NAP tries to move those feature vectors together that belong to same class but different loading conditions. Projection matrix $P$ was calculated by using Eq. (4) and it was multiplied with IFV to get transformed features. Transformed features were used with SVM classifier and classification accuracy was improved significantly as compared to baseline models. Performance of system dependent diagnosis models are compared in Table 2.

Table 3. Performance comparison of load independent and system dependent fault diagnosis models (in \% accuracy)

\begin{tabular}{ccccc}
\hline \multirow{2}{*}{ fault } & \multicolumn{3}{c}{ Baseline Models } & \multicolumn{2}{c}{$\begin{array}{c}\text { Performance Improvement } \\
\text { using NAP }\end{array}$} \\
\cline { 2 - 5 } & $3 \mathrm{kVA}$ & $5 \mathrm{kVA}$ & $3 \mathrm{kVA}$ & $5 \mathrm{kVA}$ \\
& model & model & model & model \\
\hline $\mathrm{R}$ & 72.52 & 66.43 & 95.65 & 95.75 \\
$\mathrm{Y}$ & 77.84 & 66.54 & 95.59 & 97.81 \\
$\mathrm{~B}$ & 75.17 & 90.92 & 95.89 & 95.53 \\
\hline
\end{tabular}

\subsection{Baseline system for system independent fault diagno- sis}

To develop system independent fault diagnosis model, current signals acquired for both systems at different load conditions were aggregated. In our previous work (J. Gandhi et al., 2017), baseline model for system independent fault diagnosis was implemented using wavelet domain feature extraction and support vector machine (SVM) back-end classifier. For extracting statistical features in wavelet domain, different mother wavelet functions and different levels of decomposition were experimented. We obtained optimum performance with 'db8' wavelet and 5 level of decomposition. Classification performance of baseline system for different SVM kernels are listed in Table 4. We obtained optimum classification accuracy of $65.23 \%, 78.61 \%$ and $66.2 \%$ using linear SVM. Alarm accuracy and No-Alarm accuracy are mentioned in Table 5 .

Table 4. Performance comparison of system independent baseline model for different kernels (in \% accuracy)

\begin{tabular}{ccccc}
\hline \multirow{2}{*}{ Fault } & \multicolumn{4}{c}{ SVM kernel } \\
\cline { 2 - 5 } & Linear & RBF & Polynomial & Sigmoid \\
\hline R & $\mathbf{6 5 . 2 3}$ & 69.30 & 55.34 & 55.34 \\
Y & $\mathbf{7 8 . 6 1}$ & 60.10 & 55.65 & 55.65 \\
B & $\mathbf{6 6 . 2 0}$ & 49.56 & 55.31 & 55.31 \\
\hline
\end{tabular}

Table 5. Classification performance of system independent baseline model for linear kernel (in \% accuracy)

\begin{tabular}{cccc}
\hline Fault & $\begin{array}{c}\text { Overall } \\
\text { Accuracy }\end{array}$ & $\begin{array}{c}\text { Alarm } \\
\text { Accuracy }\end{array}$ & $\begin{array}{c}\text { No-Alarm } \\
\text { Accuracy }\end{array}$ \\
\hline R & 65.23 & 63.2 & 66.86 \\
Y & 78.61 & 73.58 & 82.68 \\
B & 66.20 & 66.23 & 66.14 \\
\hline
\end{tabular}

\subsection{Removing system specific variations using NAP}

Because of SSV, performance of system independent baseline model was deteriorated as compared to system dependent baseline models. Nuisance attribute projection (NAP) can be used to remove SSV. Definition of weight matrix plays an important role while applying NAP. We used following weight matrix definition in previous work(J. Gandhi et al., 2017).

$$
W_{i j}= \begin{cases}1, & \text { if system }\left(x_{i}\right) \neq \operatorname{system}\left(x_{j}\right) \\ 0, & \text { otherwise }\end{cases}
$$

By using this weight matrix, NAP tries to move those feature vectors together that belong to different systems, however condition of the machine was not considered in this def- 


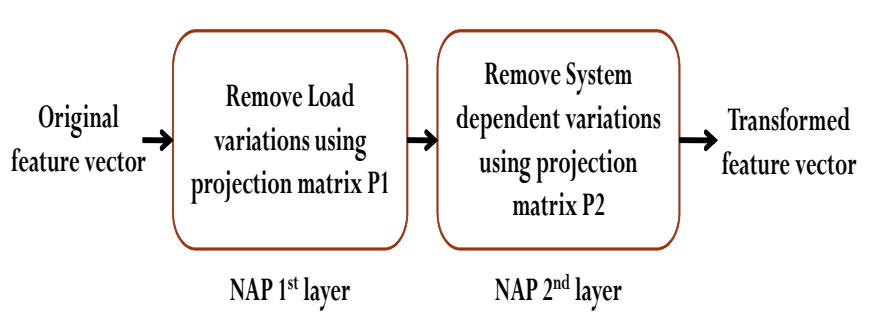

Figure 7. Stacking of NAP layers LSS-NAP

inition. Hence feature vectors belonging to different classes and different systems were also moved together, and classification performance was not improved much. This approach is referred as system NAP (S-NAP). In this work, we have incorporated condition of the machine along with system ratings in weight definition, which is mentioned below.

$$
W_{i j}= \begin{cases}1, & \text { if class }\left(x_{i}\right)=\operatorname{class}\left(x_{j}\right) \text { and } \\ & \text { system }\left(x_{i}\right) \neq \operatorname{system}\left(x_{j}\right) \\ 0, & \text { otherwise }\end{cases}
$$

By using this weight matrix, NAP tries to move those feature vectors together that belong to same condition but different systems. Hence feature vectors belonging to different classes and different systems were not moved together. This NAP approach is referred as extended system NAP (ES-NAP).

Projection matrix P was created by using Eq. (4) and used for transforming IFV. Transformed features were used with SVM back-end classifier. For ES-NAP, classification accuracy of $88.05 \%, 93.56 \%$ and $91.12 \%$ was achieved for $\mathrm{R}$, $\mathrm{Y}$ and B fault models respectively as compared to $84.86 \%$, $86.92 \%$ and $78 \%$ in S-NAP.

Since extracted features also contain LSV, effect of SSV and LSV should be reduced from IFV to have robust system independent fault diagnosis. In this work, we experimented stacked NAP and single stage NAP approaches to project out multiple nuisance attributes.

\subsection{Removing load specific and system specific variations using stacked NAP}

We experimented stacking of NAP layers in two ways: loadsystem stacked NAP (LSS-NAP) and system-load stacked NAP (SLS-NAP). In LSS-NAP, we removed LSV in first layer and SSV in second layer, which is shown graphically in Figure 7. In SLS-NAP, SSV is removed in first layer and LSV is removed in second layer, which is shown graphically in Figure 8. Definition of weight matrices to project out LSV and SSV are mentioned in Eq. (7) and Eq. (9) respectively.

In both methods, projection matrix $P_{1}$ was calculated using NAP in first layer and transformed features were obtained by

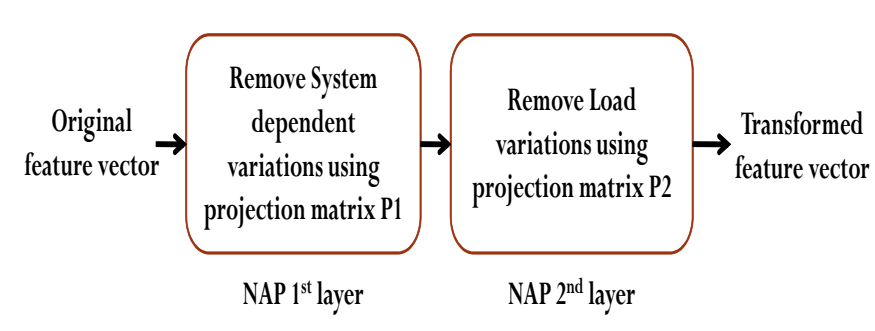

Figure 8. Stacking of NAP layers SLS-NAP

multiplying IFV with $P_{1}$. These transformed features from the first layer were used as an input to second layer. Projection matrix $P_{2}$ was calculated in second layer and multiplied with first layer transformed features to obtain final transformed features. Using LSS-NAP, classification accuracy of $89.01 \%, 94.53 \%$ and $93.58 \%$ was achieved, whereas using SLS-NAP, classification accuracy of $89.22 \%, 94.67 \%$ and $94.59 \%$ was achieved for R, Y and B fault models respectively.

\subsection{Removing load specific and system specific variations using single stage NAP}

In this approach, LSV and SSV were removed simultaneously using composite weight matrix, which is weighted sum of two NAP weight matrices responsible for removing LSV and SSV respectively. This NAP approach is referred as composite NAP (C-NAP).

$$
W=\alpha * W_{\text {load }}+\beta * W_{\text {system }}
$$

Definition of $W_{\text {load }}$ and $W_{\text {system }}$ is specified in (7) and (9) respectively. Different values of $\alpha$ and $\beta$ were experimented in range of 0 to 1 with step size of 0.1 and classification results were compared. We obtained optimum classification accuracy using $\alpha=1$ and $\beta=1$. Using these values in above equation, composite weight matrix was created and projection matrix $P$ was found by using Eq. (4). IFV was multiplied with projection matrix $P$ and used as an input to SVM classifier. Using C-NAP, classification accuracy of $89.07 \%$, $94.37 \%$ and $93.90 \%$ was achieved for R, Y and B fault models respectively. Classification accuracy of all NAP experiments for system independent fault diagnosis are compared in Table 6.

Table 6. Performance comparison of different NAP approaches for system independent fault diagnosis model (in \% accuracy)

\begin{tabular}{cccccc}
\hline Fault & S-NAP (J. Gandhi et al., 2017) & ES-NAP & LSS-NAP & SLS-NAP & C-NAP \\
\hline R & 84.86 & 88.05 & 89.01 & 89.22 & 89.07 \\
Y & 86.92 & 93.56 & 94.53 & 94.67 & 94.37 \\
B & 78.00 & 91.12 & 93.58 & 94.59 & 93.90 \\
\hline
\end{tabular}

From these results (Table 6), we can see that SLS-NAP outperforms as compared to other approaches. Table 7 lists the 
alarm accuracy and no-Alarm accuracy of SLS-NAP model.

Table 7. Classification performance of SLS-NAP model (in $\%$ accuracy)

\begin{tabular}{cccc}
\hline Fault & $\begin{array}{c}\text { Overall } \\
\text { Accuracy }\end{array}$ & $\begin{array}{c}\text { Alarm } \\
\text { Accuracy }\end{array}$ & $\begin{array}{c}\text { No-Alarm } \\
\text { Accuracy }\end{array}$ \\
\hline R & 89.22 & 83.97 & 93.45 \\
Y & 94.67 & 91.37 & 97.34 \\
B & 94.59 & 90.78 & 97.67 \\
\hline
\end{tabular}

\section{Conclusion}

In this work, we proposed concept of system independent fault diagnosis model for condition-based maintenance (CBM). This unified approach can be useful in industry environment, where single model can be used to detect faults in machines with different power ratings. To verify proposed approach, we used two fault injection capable synchronous generators with power rating of $3 \mathrm{kVA}$ and $5 \mathrm{kVA}$ as different systems. Statistical features were extracted from raw current signals using DWT. Extracted features were used with support vector machine (SVM) for fault classification.

First, we experimented for the load dependent fault diagnosis of synchronous generator. Since same loading condition and same capacity generator were used for training and testing, we obtained good classification performance. Subsequently, load independent fault diagnosis models were developed for each machine separately. However, classification performance was deteriorated due to load specific variations (LSV) in input feature vector (IFV), which were considered as a nuisance. In this work, we experimented with nuisance attribute projection (NAP) algorithm to remove LSV from the IFV and the performance was improved significantly for load independent fault diagnosis.

We then experimented for system independent fault diagnosis by using the data from $3 \mathrm{kVA}$ and 5 KVA generators. Classification accuracy of $65.23 \%, 78.61 \%$ and $66.20 \%$ was obtained for R, Y and B baseline fault models respectively. Since features extracted for two systems contain system specific variations (SSV) and LSV, classification performance was deteriorated as compared to load dependent and system dependent fault models. The nuisance attributes, LSV and SSV, were removed by using stacked NAP and single stage NAP (C-NAP) approaches. By removing SSV in first layer and LSV in second layer using stacked NAP approach (SLS-NAP), we obtained best classification performance and improved the classification accuracy by $23.99 \%, 16.06 \%$ and $28.39 \%$ for three fault models as compared to baseline models. Our experiments and results show that SLS-NAP has the potential in minimizing the system dependent factors from the input feature space for improving the performance of system indepen- dent fault diagnosis.

\section{ACKNOWLEDGMENT}

Fabrication of fault injection capable synchronous generators were carried out at M/S. Bharath Electric Motors, Coimbatore. The authors would like to thank National Program on Micro and Smart Systems (NPMASS), Aeronautical Development Agency (ADA), Bangalore, India for their financial support.

\section{BIOGRAPHIES}

Jeet Gandhi received the M.Tech. degree in Automotive Electronics from Amrita University, Coimbatore, India in 2017. His research areas include predictive maintenance, computer vision, Autonomous driving, machine learning, and deep learning.

R. Gopinath is currently pursuing $\mathrm{PhD}$ at Machine Intelligence Research Laboratory, Department of Electronics and Communication Engineering, Amrita Vishwa Vidyapeetham, Coimbatore. His areas of research interests are condition monitoring of electrical machines, vibration analysis, and machine learning.

C. Santhosh Kumar received the M.Phil degree in Computer Speech and Language Processing from University of Cambridge, United Kingdom in 1989. From 1986 to 1993, he was with Transmission R\&D, Indian Telephone Industries Ltd., Bangalore developing systems for low bit rate coding of speech. In 1993, he was with IPC Corporation Ltd., Singapore working on multimedia solutions for speech and video. From 1994 to 1999, he was with Institute of Systems Science (ISS) later known as Kent Ridge Digital Labs (KRDL), National University of Singapore, Singapore. He was responsible for development of on-line and off-line handwriting recognition systems, and small, medium, and large vocabulary speech recognition systems for Mandarin Chinese. From 1999 to 2001, he was with Lernout \& Hauspie (L\&H) Asia Pacific, working on localization of the L\&H speech recognition technology. Since 2001, he has been teaching at the Department of Electronics and Communication Engineering, Amrita Vishwa Vidyapeetham. In 2011, he completed his Ph.D from Amrita Vishwa Vidyapeetham, with the thesis focusing on multi-lingual acoustic modeling for the Indian languages. He was a Visiting Researcher to Faculty of Information Technology, Brno University of Technology, Czech Republic in 2007. In 2009, he served as a Visiting Researcher to School of Computer and Electrical Engineering, University of Auckland, New Zealand, and School of Electrical Engineering, University of New South Wales, Sydney, Australia. He is currently leading the research activities in Machine Intelligence Research Lab of Amrita School of Engineering, Coimbatore. His areas of research interest are spoken language processing, machine fault diagnosis, and the development of 
biomedical systems to make affordable health-care systems for rural India.

\section{REFERENCES}

Awadallah, M. A., \& Morcos, M. M. (2003). Application of ai tools in fault diagnosis of electrical machines and drives-an overview. IEEE Transactions on energy conversion, 18(2), 245-251.

Bendjama, H., Bouhouche, S., \& Boucherit, M. S. (2012). Application of wavelet transform for fault diagnosis in rotating machinery. International Journal of Machine Learning and Computing, 2(1), 82.

Bessam, B., Menacer, A., Boumehraz, M., \& Cherif, H. (2017). Wavelet transform and neural network techniques for inter-turn short circuit diagnosis and location in induction motor. International Journal of System Assurance Engineering and Management, 8(1), 478488.

Dash, R. N., Subudhi, B., \& Das, S. (2010). Induction motor stator inter-turn fault detection using wavelet transform technique. In Industrial and information systems (iciis), 2010 international conference on (pp. 436-441).

Daubechies, I. (1988). Orthonormal bases of compactly supported wavelets. Communications on pure and applied mathematics, 41(7), 909-996.

Erişti, H., Uçar, A., \& Demir, Y. (2010). Wavelet-based feature extraction and selection for classification of power system disturbances using support vector machines. Electric power systems research, 80(7), 743-752.

Gandhi, A., Corrigan, T., \& Parsa, L. (2011). Recent advances in modeling and online detection of stator interturn faults in electrical motors. IEEE Transactions on Industrial Electronics, 58(5), 1564-1575.

Gandhi, J., Gopinath, R., \& Kumar, C. S. (2017). Nuisance attribute projection for system independent fault diagnosis of synchronous generator. In Wireless coтmunication, signal processing and networking (wispnet), 2017 international conference on (pp. 563-568).

Gopinath, R., Kumar, C. S., \& Ramachandran, K. (2016). Scalable fault models for diagnosis of synchronous generators. International Journal of Intelligent Systems Technologies and Applications, 15(1), 35-51.

Gopinath, R., Kumar, C. S., Ramachandran, K., Upendranath, V., \& Kiran, P. S. (2016). Intelligent fault diagnosis of synchronous generators. Expert Systems with Applications, 45, 142-149.

Gopinath, R., Kumar, C. S., Vishnuprasad, K., \& Ramachandran, K. (2015). Feature mapping techniques for improving the performance of fault diagnosis of synchronous generator. International Journal of Prognostics and Health Management, 6(2), 12.

Gopinath, R., Nambiar, T., Abhishek, S., Pramodh, S. M.,
Pushparajan, M., Ramachandran, K., ... Thirugnanam, R. (2013). Fault injection capable synchronous generator for condition based maintenance. In Intelligent systems and control (isco), 2013 7th international conference on (pp. 60-64).

Jardine, A. K., Lin, D., \& Banjevic, D. (2006). A review on machinery diagnostics and prognostics implementing condition-based maintenance. Mechanical systems and signal processing, 20(7), 1483-1510.

Krishna, V., Chendur, P. P., Abhilash, P., Abraham, R. T., Gopinath, R., \& Kumar, C. S. (2016). Improving the performance of wavelet based machine fault diagnosis system using locality constrained linear coding. In The international symposium on intelligent systems technologies and applications (pp. 951-964).

Nandi, S., Toliyat, H. A., \& Li, X. (2005). Condition monitoring and fault diagnosis of electrical motorsa review. IEEE transactions on energy conversion, 20(4), 719729.

Neti, P., \& Nandi, S. (2009). Stator interturn fault detection of synchronous machines using field current and rotor search-coil voltage signature analysis. IEEE Transactions on Industry Applications, 45(3), 911-920.

Rajeswari, R., \& Kamaraj, N. (2007). Diagnosis of inter turn fault in the stator of synchronous generator using wavelet based anfis. World Academy of Science, Engineering and Technology, 36, 203-209.

Siddique, A., Yadava, G., \& Singh, B. (2005). A review of stator fault monitoring techniques of induction motors. IEEE transactions on energy conversion, 20(1), 106114.

Solomonoff, A., Campbell, W. M., \& Boardman, I. (2005). Advances in channel compensation for svm speaker recognition. In Acoustics, speech, and signal processing, 2005. proceedings.(icassp'05). ieee international conference on (Vol. 1, pp. I-629).

Solomonoff, A., Campbell, W. M., \& Quillen, C. (2007). Nuisance attribute projection. Speech Communication, $1-73$.

Štruc, V., Vesnicer, B., Mihelič, F., \& Pavešič, N. (2010). Removing illumination artifacts from face images using the nuisance attribute projection. In Acoustics speech and signal processing (icassp), 2010 ieee international conference on (pp. 846-849).

Tome, P., Vera-Rodriguez, R., Fierrez, J., \& Ortega-García, J. (2012). Variability compensation using nap for unconstrained face recognition. In Distributed computing and artificial intelligence (pp. 129-139). Springer.

Wang, C., Liu, X., \& Chen, Z. (2014). Incipient stator insulation fault detection of permanent magnet synchronous wind generators based on hilbert-huang transformation. IEEE Transactions on Magnetics, 50(11), 1-4.

Wu, J.-D., \& Liu, C.-H. (2008). Investigation of engine fault diagnosis using discrete wavelet transform and neu- 
ral network. Expert Systems with Applications, 35(3), $1200-1213$.

Yan, R., Gao, R. X., \& Chen, X. (2014). Wavelets for fault diagnosis of rotary machines: A review with applications. Signal Processing, 96, 1-15.

Yifrach, A., Novoselsky, E., Solewicz, Y. A., \& Yitzhaky, Y. (2016). Improved nuisance attribute projection for face recognition. Pattern Analysis and Applications, 19(1), 69-78.

Yin, S., Ding, S. X., Xie, X., \& Luo, H. (2014). A review on basic data-driven approaches for industrial process monitoring. IEEE Transactions on Industrial Electronics, 61(11), 6418-6428. 